\title{
Patients' Satisfaction and Associated Factors Among Outpatient Department at Wolaita Sodo University Teaching Hospital, Southern Ethiopia: A Cross Sectional Study
}

\author{
Getu Gamo Sagaro ${ }^{1, ~ *, ~ A l e m a y e h u ~ W o r k u ~ Y a l e w ~}{ }^{2}$, Mengistu Meskele Koyira ${ }^{1}$ \\ ${ }^{1}$ School of Public Health, College of Health Sciences and Medicine, Wolaita Sodo University, Wolaita Sodo, Ethiopia \\ ${ }^{2}$ School of Public Health, College of Health Sciences, Addis Ababa University, Addis Ababa, Ethiopia
}

Email address:

gamogetu48@gmail.com (G. G. Sagaro), alemayehuwy@yahoo.com (A. W. Yalew), mengistu77@gmail.com (M. M. Koyira)

\section{To cite this article:}

Getu Gamo Sagaro, Alemayehu Worku Yalew, Mengistu Meskele Koyira. Patients' Satisfaction and Associated Factors among Outpatient Department at Wolaita Sodo University Teaching Hospital, Southern Ethiopia: A Cross Sectional Study. Science Journal of Clinical Medicine.Vol. 4, No. 5, 2015, pp. 109-116. doi: 10.11648/j.sjcm.20150405.16

\begin{abstract}
Background: Asking patients what they think about the care and treatment they have received is an important step towards improving the quality of care, and to ensuring that local health services are meeting patients' needs and expectations. Therefore, identifying factors affecting patient satisfaction at local level is a paramount importance to plan and monitor the utilization of health care services. This study aimed to measure level of satisfaction and associated factors among patients receiving health services in outpatient departments at Wolaita Sodo University Teaching Hospital, Southern Ethiopia. Method: Facility based cross sectional study was conducted from February 1 - 28, 2015 on 421 health care service users using systematic random sampling technique. Data was collected using interviewer administrated pre-tested structured questionnaire and entered into EPI Info version 3.5.1 and then exported and analyzed using SPSS version 20. Variables having $\mathrm{p}<0.2$ at bivariate analysis were fitted to multivariate analysis. OR, P-value and 95\% CI were computed to show the association of variables $(\mathrm{p}<0.05)$. Result: Among the total sample size $(\mathrm{n}=421)$ four hundred fifteen adult patients were exit interviewed, giving a response rate of $98.6 \%$, of which 211 (50.8) were medicine clinic. Overall patient satisfaction with the health care service provided at OPD clinics of the hospital was 54.2\% at 95\% CI (49.4\% - 59\%). The respondents who perceived medium empathy by the provider were found to be more satisfied as compared those who were perceived low empathy (AOR : 2.39(1.32-4.33). Moreover, respondents who reported that the waiting area was clean were more satisfied as compared to those who reported waiting area not clean (AOR: 2.53(1.44-4.47). In addition, respondents waited less than or equal to 30 minutes in waiting area preceding consultation were to be more satisfied than those who were waited 60 minutes and above (AOR: 3.16(1.37-7.25).Conclusion: The overall satisfaction of outpatient health care services provided at Wolaita Sodo University Teaching Hospital was low. Hospital managements and service providers should give attention to reduce waiting time, ensure good interactions with patients, improve drug availability and maintain privacy of patients in order to improve the level of satisfaction of patients.
\end{abstract}

Keywords: Patient Satisfaction, Outpatient, Patient, Hospital

\section{Introduction}

Patient satisfaction is the fundamental importance as a measure of the quality of care because it gives information on the provider's success in meeting client values and expectations, matters on which the client is the ultimate authority (1). In recent decades, determining the level of patient satisfaction has been found to be the most useful tool for getting patients' views on how to provide care. Generally, patients are the best source of information on both quality and quantity of health care services provided and patients' views are determining factors in planning and evaluating satisfaction (2).

Healthcare facility performance can be best assessed by measuring the level of patient's satisfaction. Measurement of patients' satisfaction is increasingly playing important role in the growing push towards accountability among healthcare providers, thus some view it as an established indicator of quality of care (3) and studies have shown that satisfied patients are more likely to comply with prescribed treatment 
and advice from doctors. In addition, they are also more likely to return for additional care when necessary and may be more willing to pay for services, thereby increasing revenue (4).

The outpatient department in any hospital considered to be shop window of the hospital, hence, there are various problems faced by the patients in outpatient department clinics, such as overcrowding, delay in consultation, lack of proper guidance and so on, which leads the patients to be dissatisfied (5).

Studies conducted in Out Patient Departments (OPD) of different hospitals in Ethiopia have observed long waiting time during registration, visiting of doctors after registration, privacy of examination room, laboratory procedures and revisiting of the doctor for evaluation with laboratory results, failure to obtain prescribed drugs and supplies from the hospitals' pharmacies and inadequate information provision were the frequently faced problems affecting utilization leading to dissatisfaction $(6,7,8)$.

In Ethiopia, the potential health service coverage based on availability of health services is estimated $86.7 \%$. But, Utilization of services for the country is as low as 0.32 (9).Such, low rate could entail low availability including basic service capacity standards, affordability and quality of services (10). Therefore, patient satisfaction is an important outcome measure for medical services. In addition, data on patient satisfaction are the key indicators for the quality of care and treatment delivered by the physicians, paramedical staff and the hospital as a whole (11). The study conducted in developing countries including Ethiopia by World Bank showed that the level of patient satisfaction was low (12).

Asking patients what they think about the care and treatment they have received is an important step towards improving the quality of care and knowing the predictors of patient satisfaction is very important to provide quality healthcare services and the utilization of health services in public hospitals (13). Hence, Wolaita Sodo University is undertaking different activities to improve the quality of health care service delivery. But, the level of patient's satisfaction with the Wolaita Sodo University Teaching Hospital outpatient health care services is not studied so far.

The aim of this study is to measure the level of patients' satisfaction after a visit with outpatient department health services and factors associated with it in Wolaita Sodo University Teaching Hospital (WSUTH). Hence, this study provides evidence - based information to improve health service delivery and also, helpful to fill gaps which ultimately contribute to desirable quality of outpatient services in the hospital and enhancing the level of patients' satisfaction.

\section{Methods}

\subsection{Study Setting and Period}

The study was conducted in Wolaita Sodo University Teaching Hospital in OPD regular working hours from
February $1-28$ / 2015. The hospital is found in South Nations Nationalities and People Region States (SNNPRs), Ethiopia. It is located in Sodo Town of Wolaita Zone, SNNPRS which is $380 \mathrm{~km}$ away from the national capital Addis Ababa and $170 \mathrm{~km}$ far from the regional capital, Hawassa. The hospital is serving more than 2 million people in the catchment area in all four major departments and other units since 1928.

\subsection{Study Design, Participants, Sample Selection}

A facility based cross sectional study design was conducted. The study includes both adult greater than or equal to 18 years and age under 18 , their parents or caretakers were interviewed in four outpatient department clinics (Medicine, surgery, pediatric and obstetrics and gynecology).

Sample size was calculated by using single population proportion formula with the following assumptions: Proportion of patients satisfied with hospital care services to be $80.1 \%$, according to a study done in Hawassa University Teaching Hospital (7), margin of error 4\%, Confidence level of $95 \%$, Considering $10 \%$ non-response rate. The calculated sample size was 421. The total sample size was proportionately allocated for each service clinics depending on the average number of patients who visited the outpatient department clinics one month prior to the start of the study. Then study participants were identified by systematic random sampling method. In addition, Simple random sampling was done for the first patients to get the starting point. Thereafter, depending on sampling interval patients' coming to the clinics to be enrolled in the study until the required sample size was obtained.

Data was collected using pre-tested structured questionnaires by exit interview in four confidential rooms. The questionnaire was adapted from related literatures with slight modification made with the objective of this particular study and to fit with local context. The questionnaire in the beginning was prepared in English and then translated into Amharic and back to English to ensure consistency, but finally interview was administered by four clinical nurses who can speak Wolaita and Amharic fluently.

\subsection{Data Management and Analysis}

Data was cheeked and cleaned for completeness and accuracy, and entered into EPI Info version 3.5.1 and then exported and analyzed using SPSS version 20. Bi-variate analysis was done and variables with p-value less than 0.2 were included in multiple logistic regressions analysis to control confounder and identify the most significant predictor of outcome variables. Respondents rated their satisfaction with various aspects of services provided, using a five-point Likert scale contains five items (1-Strongly dissatisfied, 2dissatisfied, 3-neutral, 4-satisfied, and 5-Strongly satisfied). Responses for each five item was summed and transformed to give an overall satisfaction score from 0 to100 percentage for each items used in the scales(14),Descriptive statistics 
was computed for the study variables and frequency distribution tables were used to describe most of the findings.

\subsection{Operational Definitions}

Level of patient satisfaction: -all five measuring items in the scale to measure satisfaction together yield a maximum score of 25 and a minimum score 5. Satisfaction level was measured by the responses for each five item was summed and transformed to give an individual level satisfaction score from 0 to 100 percentage for each items used as percentage mean score.

Overall satisfaction level:75\%and above response rate of the five satisfaction measuring items were categorized as "satisfied" and those who were satisfied in less than $75 \%$ of the five satisfaction measuring items were categorized as "unsatisfied" (15).

Consultation and Relational Empathy (CARE): Within the CARE tool patients were asked 10 questions to rate different aspects of empathy. Each question was scored on an ordinal scale from 1 (poor) to 5 (excellent). Scores are added, with the maximum possible score being 50 and the minimum 10 . Perceived empathy was categorized as indicating low (1024), medium (25-37) and high (38-50) (16).

\subsection{Ethical Consideration}

The study was approved by Ethics review board of Wolaita Sodo University, school of public health, College of Health Science and Medicine. Support letter and permission was obtained from Wolaita Sodo University Teaching Referral Hospital. Informed verbal consent was obtained from each client after explaining the objective of the study. Clients were assured that refusal to participate does not affect their care and services they are obtaining. The client's privacy and confidentiality was assured.

\section{Result}

\subsection{Socio-Demographic Characteristics of Respondents}

Among the total sample size $(n=421)$ four hundred fifteen adult patients' were exit interviewed, giving a response rate of $98.6 \%$, of which 211 (50.8) were medicine clinic. The mean (SD) age of the respondents was $34.17 \pm 11.67$ (range $=$ $18-75 y e a r s)$ and $153(36.9 \%)$ of the respondents were between the age category of $18-27$ years. More than two third of respondents were married $(75.2 \%)$ Table 1.

Table1. Socio-demographic characteristics of respondents with health care service provided in WSUTH, February $2015(n=415)$.

\begin{tabular}{lll}
\hline Socio demographic variables & Number & percent \\
\hline Age category & & \\
$18-27$ & 153 & 36.9 \\
$28-37$ & 128 & 30.8 \\
$38-47$ & 73 & 17.6 \\
$48^{+}$ & 61 & 14.7 \\
Sex & & \\
Male & 234 & 56.4 \\
\hline
\end{tabular}

\begin{tabular}{|c|c|c|}
\hline Socio demographic variables & Number & percent \\
\hline Female & 181 & 43.6 \\
\hline \multicolumn{3}{|l|}{ Marital Status } \\
\hline Single & 95 & 22.9 \\
\hline Married & 312 & 75.2 \\
\hline Divorced/separated & 3 & 0.7 \\
\hline Widowed & 5 & 1.2 \\
\hline \multicolumn{3}{|l|}{ Religion } \\
\hline Protestant & 288 & 69.4 \\
\hline Catholic & 17 & 4.1 \\
\hline Orthodox & 90 & 21.7 \\
\hline Other & 20 & 4.8 \\
\hline \multicolumn{3}{|l|}{ Ethnicity } \\
\hline Wolaita & 333 & 80.2 \\
\hline Kamabata & 13 & 3.1 \\
\hline Gamo & 15 & 3.6 \\
\hline Other & 54 & 13 \\
\hline \multicolumn{3}{|l|}{ Educational Status } \\
\hline Not attended school & 67 & 16.1 \\
\hline In or completed 1 st to 4 th grade & 44 & 10.6 \\
\hline In or completed $5^{\text {th }}$ to $8^{\text {th }}$ grade & 87 & 21 \\
\hline In or completed $9^{\text {th }}$ to $10^{\text {th }}$ grade & 74 & 17.8 \\
\hline In or completed $11^{\text {th }}$ to $12^{\text {th }}$ grade & 31 & 7.5 \\
\hline In or completed college or university & 112 & 27 \\
\hline \multicolumn{3}{|l|}{ Occupational status } \\
\hline Farmer & 84 & 20.2 \\
\hline House wife & 105 & 25.3 \\
\hline Student & 39 & 9.4 \\
\hline Employee & 103 & 24.8 \\
\hline Merchant & 57 & 13.7 \\
\hline Other & 27 & 6.5 \\
\hline \multicolumn{3}{|l|}{ Family monthly income } \\
\hline$<750$ birr & 281 & 67.7 \\
\hline$\geq 750$ birr & 134 & 32.3 \\
\hline
\end{tabular}

\subsection{Institutional Aspects and Pattern of Visit}

Table 2. Institutional aspects and pattern of visit of outpatient department clinics with health services provided at WSUTH, February 2015 $(n=415)$.

\begin{tabular}{lll}
\hline Variables & Number & percent \\
\hline Waiting time in registration process & 101 & 24.3 \\
Long & 161 & 138.8 \\
Fair & 153 & 36.9 \\
Short & & \\
Registration process was done timely & 334 & 80.5 \\
Agree & 81 & 19.5 \\
Disagree & & \\
Waiting time to visit service provider (in minutes) & 167 & 40.2 \\
<30 & 184 & 44.3 \\
$31-60$ & 64 & 15.4 \\
60+ & & \\
Laboratory test/x-ray/ultrasound examination ordered & 238 & 57.3 \\
Yes & 177 & 42.7 \\
No & & \\
Laboratory/x-ray/ultrasound result waiting time & 108 & 45.4 \\
Long & 75 & 31.5 \\
Fair & 55 & 23.1 \\
Short & & \\
Availability of prescribed drugs & 267 & 64.3 \\
All & 127 & 30.6 \\
Some & 21 & 5.1 \\
None & & \\
Waiting time for receiving medicines & & \\
\hline & & \\
\hline
\end{tabular}




\begin{tabular}{lll}
\hline Variables & Number & percent \\
\hline Long & 70 & 17.3 \\
Fair & 147 & 36.4 \\
Short & 187 & 46.3 \\
Type of visit & & \\
New & 144 & 34.7 \\
Repeated/Follow-up & 171 & 65.3 \\
Frequency of visit in the last 12 months & & \\
One & 144 & 34.7 \\
Two & 66 & 15.9 \\
Three & 95 & 22.9 \\
>=four & 110 & 26.5 \\
Knowing provider & & \\
Very well & 16 & 3.9 \\
Well & 13 & 3.1 \\
Know little bit & 90 & 21.7 \\
Not at all & 296 & 71.3 \\
Privacy of consultation room appropriate & 355 & 85.5 \\
Privacy respected during consultation & 332 & 80 \\
Clean waiting area & 204 & 49.2 \\
Clean consultation room & 368 & 88.7 \\
Interviewed language you understand & 399 & 96.1 \\
\hline
\end{tabular}

Among the total respondents, 271 (65.3\%) respondents were repeated or follow - up visitors, while 144 (34.7\%) were new visitors. Of the respondent's $110(26.5 \%)$ respondents were visited previous OPD clinics for greater than or equal to four times. In this study, the mean (SD) waiting time to see service provider preceding consultation in waiting area was $56.78 \pm 65.93$ minutes. More than nine in ten $(96.1 \%)$ of the respondents reported that they were interviewed in the language they understand. Nearly two third (64.3\%) of the respondents were reported to have got all prescribed drugs from hospital pharmacy. Regard to privacy, 83 (20\%) of the respondents claimed that their privacy was not respected during consultation but $355(85.5 \%)$ of the respondents felt that the consultation room provide adequate privacy. However, $211(50.8 \%)$ of the respondents reported that the waiting area was not clean (Table 2).

\subsection{Interaction with the Health Care Provider}

Table 3. Perceived patients and provider interaction variable of patient satisfaction with OPD clinics services at WSUTH, Southern Ethiopia, February 2015(n=415).

\begin{tabular}{lll}
\hline Variables & Number & percent \\
\hline Provider told the name of your illness & 285 & 68.7 \\
Provider told the cause of your illness & 184 & 44.3 \\
Provider told to return if it gets worse & 294 & 70.8 \\
$\begin{array}{l}\text { Provider told enough about your treatment } \\
\text { Provider told you ways of preventing future }\end{array}$ & 220 & 53 \\
recurrence & 220 & 53 \\
Told your private issues & 346 & 83.4 \\
$\begin{array}{l}\text { Consultation duration(in minutes) } \\
<6.10\end{array}$ & 310 & 74.7 \\
$\geq 6.10$ & 105 & 25.3 \\
Perceived consultation duration & & \\
Long & 35 & 8.4 \\
Fair & 220 & 53 \\
Short & 160 & 38.6 \\
Perceived empathy & & \\
Low & 147 & 35.4 \\
Medium & 242 & 58.3 \\
High & 26 & 6.3 \\
\hline
\end{tabular}

Among the total number of the respondents, more than two third $(68.7 \%)$ of the respondents reported that the provider told the name of their illness while more than half $(53 \%)$ of the respondents were given advice on how to prevent the reoccurrence of their illness. Concerning to duration stay with service provider, the mean (SD) time of consultation duration was $6.10 \pm 4.12$ minutes (range $=3-20$ minutes). Regarding perceived empathy $242(58.3 \%)$ have medium, $147(35.4 \%)$ have low, while the remaining $26(6.3 \%)$ have high (Table3).

\subsection{Respondents Score on Satisfaction Measuring Items}

Among the total respondents $(n=415) ; 64.8 \%$ were responded as "satisfied" with instructions given by the doctor on investigations/prescriptions; while $14(3.4 \%)$ of them were strongly dissatisfied. Majority (68\%) were satisfied with the amount of time spent with the doctor; while 15(3.6\%) were strongly dissatisfied (Table4).

\subsection{Determining Patient Satisfaction}

The proportion of respondents who were satisfied with health care service provided in this study was $54.2 \%$ at $95 \%$ CI $(49.4 \%$ - 59\%) computed from satisfaction measuring items (Figure 1)

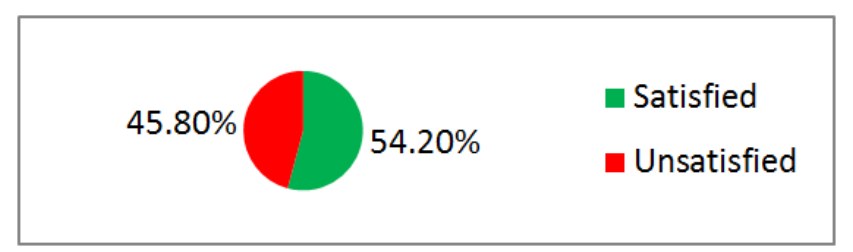

Fig. 1. Diagrammatic presentation of patient satisfaction with OPD health care service in Wolaita Sodo University Teaching Hospital, 2015.

\subsection{Factors Associated with Patient Satisfactions}

Binary logistic regression was conducted using bi-variate analysis for socio-demographic variables, institutional aspects and pattern of visit and interaction with health care service provider variables. Those variables which were with $\mathrm{p}$-values less than 0.2 at bi-variate logistic regression were included in multiple logistic regressions.

In multivariate analysis, factors that remained statistically significant with patient satisfaction were perceived empathy (AOR 2.39, 95\%CI: 1.32-4.33), availability of prescribed drugs in hospital pharmacy (AOR 0.44, 95\% CI:0.25-0.76), privacy respected during consultation (AOR 4.08, 95\% CI:2.08-8.01), cleanliness of waiting area (AOR 2.53, 95\% CI:1.44-4.47), cleanliness of consultation room (AOR 2.42, 95\% CI:1.07-5.50), interviewed by the language you understand (AOR 6.27, 95\% CI:1.16-33.78), advice given how to prevent reoccurrence of disease (AOR 1.80, 95\% CI:1.06-3.04), waiting time to service provider less than or equal to 30 minutes preceding consultation in waiting area (AOR 3.16, 95\% CI:1.37-7.25) and payment status (AOR 2.06, 95\% CI:1.02-4.17) of the respondents (Table 5). 
Table 4. Respondents level of satisfaction with OPD clinics different components of health care services at WSUTH, Southern Ethiopia, February $2015(n=415)$.

\begin{tabular}{|c|c|c|c|c|c|}
\hline Items & $\begin{array}{l}\text { Strongly } \\
\text { dissatisfied N (\%) }\end{array}$ & $\begin{array}{l}\text { Dissatisfied } \\
\mathrm{N}(\%)\end{array}$ & $\begin{array}{l}\text { Neutral } \\
\text { N (\%) } \\
\end{array}$ & $\begin{array}{l}\text { Satisfied } \\
\text { N (\%) }\end{array}$ & $\begin{array}{l}\text { Strongly satisfied } \\
\mathbf{N}(\%)\end{array}$ \\
\hline I would come back to this hospital & $11(2.7)$ & $53(12.8)$ & $13(3.1)$ & $288(69.4)$ & $50(12)$ \\
\hline I would send my friends or relatives to this hospital & $15(3.6)$ & $40(9.6)$ & $50(12)$ & $275(66.3)$ & $35(8.4)$ \\
\hline $\begin{array}{l}\text { I am satisfied with instructions given by the doctor on } \\
\text { investigations/prescriptions }\end{array}$ & $14(3.4)$ & $52(12.5)$ & $46(11.1)$ & $269(64.8)$ & $34(8.2)$ \\
\hline I am satisfied with the amount of time spent with the doctor & $15(3.6)$ & $72(17.3)$ & $17(4.1)$ & $282(68)$ & 29(7) \\
\hline $\begin{array}{l}\text { I am satisfied with the condition (comfort, privacy etc.) of the } \\
\text { consulting room }\end{array}$ & $10(2.4)$ & $62(14.9)$ & $11(2.7)$ & $302(72.8)$ & $30(7.2)$ \\
\hline
\end{tabular}

Table 5. Bi-variate and multivariate analysis showing factors associated with patient satisfaction WSUTH, Southern Ethiopia, February 2015(n=415).

\begin{tabular}{|c|c|c|c|c|}
\hline \multirow{2}{*}{ Explanatory variables } & \multicolumn{2}{|c|}{ Patient satisfaction } & \multirow{2}{*}{$\operatorname{COR}(95 \% \mathrm{CI})$} & \multirow{2}{*}{$\operatorname{AOR}(95 \% \mathrm{CI})$} \\
\hline & SatisfiedN (\%) & UnsatisfiedN (\%) & & \\
\hline \multicolumn{5}{|l|}{ Sex } \\
\hline Male & $111(49.3)$ & $123(64.7)$ & 1 & 1 \\
\hline Female & $114(50.7)$ & $67(35.3)$ & $1.89(1.27-2.80)^{* *}$ & $1.46(0.86-2.47)$ \\
\hline \multicolumn{5}{|c|}{ Waiting time to visit service provider (in minutes) } \\
\hline$<30$ & $109(48,4)$ & $58(30.5)$ & $5.20(2.74-9.85)^{* * *}$ & $3.16(1.37-7.25)^{* *}$ \\
\hline $31-60$ & $99(44)$ & $85(44.7)$ & $3.22(1.72-6.02)^{* * *}$ & $1.20(0.89-4.49)$ \\
\hline $60+$ & $17(7.6)$ & $47(24.7)$ & 1 & 1 \\
\hline \multicolumn{5}{|l|}{ Availability of prescribed drugs } \\
\hline All & $160(71.1)$ & $107(56.3)$ & 1 & 1 \\
\hline Some & $57(25.3)$ & $70(36.8)$ & $0.55(0.36-0.83)^{* *}$ & $0.44(0.25-0.76)^{* *}$ \\
\hline None & $8(3.6)$ & $13(6.8)$ & $0.41(0.17-1.03)$ & $0.43(0.088,2.112)$ \\
\hline \multicolumn{5}{|l|}{ How much did you know the provider? } \\
\hline Very well & $12(5.3)$ & $4(2.1)$ & $2.96(0.93-9.39)$ & $1.90(0.47,7.73)$ \\
\hline Well & $8(3.6)$ & $5(2.6)$ & $1.58(0.51-4.94)$ & $1.41(0.30-6.64)$ \\
\hline Know little bit & $56(24.9)$ & $34(17.9)$ & $1.63(1.00-2.63)^{*}$ & $1.80(0.93-3.46)$ \\
\hline Not at all & $149(66.2)$ & $147(77.4)$ & 1 & 1 \\
\hline \multicolumn{5}{|l|}{ Educational status } \\
\hline Not attended school & $31(13.8)$ & $36(18.9)$ & $0.89(.487-1.637)$ & $0.59(0.28-1.27)$ \\
\hline In or completed $1^{\text {st }}$ to $4^{\text {th }}$ grade & $24(10.7)$ & $20(10.5)$ & $1.24(.618-2.503)$ & $1.45(0.59-3.57)$ \\
\hline In or completed $5^{\text {th }}$ to $8^{\text {th }}$ grade & $52(23.1)$ & $35(18.4)$ & $1.54(.874-2.713)$ & $1.37(0.67-2.83)$ \\
\hline In or completed $9^{\text {th }}$ to $10^{\text {th }}$ grade & $44(19.6)$ & $30(15.8)$ & $1.52(0.84-2.75)$ & $2.00(0.93-4.33)$ \\
\hline In or completed $11^{\text {th }}$ to $12^{\text {th }}$ grade & $19(8.4)$ & $12(6.3)$ & $1.64(0.74-3.70)$ & $1.17(0.4-, 3.30)$ \\
\hline In or completed college or university & $55(24.4)$ & $57(30)$ & 1 & 1 \\
\hline \multicolumn{5}{|l|}{ Payment status } \\
\hline Paying & $184(81.8)$ & $166(87.4)$ & 1 & 1 \\
\hline Free & $41(18.2)$ & $24(12.6)$ & $1.54(0.89-2.66)$ & $2.06(1.02-4.17)^{*}$ \\
\hline \multicolumn{5}{|l|}{ Waiting time for receiving medicines } \\
\hline Long & $30(13.6)$ & $40(21.7)$ & 1 & 1 \\
\hline Fair & $88(40)$ & $59(32.1)$ & $1.99(1.12-3.54)^{*}$ & $1.48(0.70-3.12)$ \\
\hline Short & 102(46.4) & $85(46.2)$ & $1.60(0.92-2.784)$ & $1.19(0.57-2.49)$ \\
\hline \multicolumn{5}{|l|}{ Privacy respected during consultation } \\
\hline Yes & 204(90.7) & $128(67.4)$ & $4.71(2.74-8.09)^{* * *}$ & $4.08(2.08-8.01)^{* * *}$ \\
\hline \multicolumn{5}{|l|}{ waiting area clean } \\
\hline Yes & $135(60)$ & $69(36.3)$ & $2.63(1.77-3.92)^{* * *}$ & $2.53(1.44-4.47)^{* * *}$ \\
\hline No & $90(40)$ & $121(63.7)$ & 1 & 1 \\
\hline \multicolumn{5}{|l|}{ Consultation room clean } \\
\hline Yes & $212(94.2)$ & $156(82.1)$ & $3.55(1.82-6.96)^{* * *}$ & $2.42(1.07-5.50)^{*}$ \\
\hline No & $13(5.8)$ & $34(17.9)$ & 1 & 1 \\
\hline \multicolumn{5}{|l|}{ Perceived empathy } \\
\hline Low & $45(20)$ & $102(53.7)$ & 1 & 1 \\
\hline Medium & $158(70.2)$ & $84(44.2)$ & $4.26(2.75-6.62)^{* * *}$ & $2.39(1.32-4.33)^{* *}$ \\
\hline High & $22(9.8)$ & $4(2.1)$ & $12.47(4.06-8.70)^{* * *}$ & $4.28(0.99-18.57)$ \\
\hline
\end{tabular}




\begin{tabular}{|c|c|c|c|c|}
\hline \multirow{2}{*}{ Explanatory variables } & \multicolumn{2}{|c|}{ Patient satisfaction } & \multirow{2}{*}{$\operatorname{COR}(95 \% \mathrm{CI})$} & \multirow{2}{*}{$\operatorname{AOR}(95 \% \mathrm{CI})$} \\
\hline & SatisfiedN (\%) & UnsatisfiedN (\%) & & \\
\hline Yes & $223(99.1)$ & $176(92.6)$ & $8.87(1.99-39.54)^{* *}$ & $6.27(1.16-33.78)^{*}$ \\
\hline No & $2(0.9)$ & $14(7.4)$ & 1 & 1 \\
\hline \multicolumn{5}{|c|}{ Provider told you how to prevent reoccurrence of your illness } \\
\hline Yes & $146(64.9)$ & $74(38.9)$ & $2.90(1.94-4.32)^{* * *}$ & $1.80(1.06-3.04)^{*}$ \\
\hline No & $79(35.1)$ & $116(61.1)$ & 1 & 1 \\
\hline \multicolumn{5}{|c|}{ Consultation duration(in minutes) } \\
\hline$<6.10$ & $153(68)$ & $157(82.6)$ & 1 & 1 \\
\hline$\geq 6.10$ & $72(32)$ & $33(17.4)$ & $2.24(1.40-3.58)^{* *}$ & $1.62(0.88-2.96)$ \\
\hline
\end{tabular}

(*) P -value $<0.05,(* *)$ P- value $<0.01(* * *)$ P- value $<0.001$.

AOR: Adjusted odd ratio, COR: Crude odd ratio.

\section{Discussion}

The study was conducted in the OPD during the regular working hours and exclude weekends and holy days. This study showed that the overall satisfaction level of the outpatient with OPD clinics health care services provided at WSUTH was $54.2 \%$ at $95 \%$ CI $(49.4 \%$ - 59\%). This overall satisfaction level report is low compared to the report of the studies conducted in Jimma University Specialized Hospital, Hawassa University Teaching Hospital, Amhara Region Referral Hospital and Health centers in central Ethiopia, which showed $77 \%, 80.1 \%, 61.9 \%$ and $62.6 \%$ respectively $(6,7,15,17)$ but the findings comparable with studies conducted in Eastern Ethiopia and Jimma hospital, which showed $54.1 \%$ and $57.1 \%$ respectively $(18,19)$. On the other hand, this finding is higher than the reports of a study conducted in the hospitals of Amhara region and which showed that satisfaction level from $22 \%$ to $50 \%(8,20)$ and report from Tigray Zonal hospital revealed satisfaction level of $43.6 \%$ (21).The difference might be the fact that specialized teaching hospitals are equipped very well and have enough diversity of health professionals, better diagnostic facilities, health service infrastructures, and awareness of service providers of different levels that are expected to demonstrate the standard way of patient examination resulting in higher overall satisfaction level $(6,7)$. In addition, computing way of overall satisfaction level, different classification of satisfaction level, high patient load, shortage of staff, study times and design also contributed.

According to the result of present study perceived empathy is an important factor predicting the level of patient satisfaction. Furthermore as perceived empathy gets better, it's effect on patient satisfaction becomes more positive. So it is important to give attention to the effectiveness of empathy in patient - physician communication during consultation. This supports the view that perceived empathy or empathetic communication during consultation is an essential prerequisite for the delivery of quality health care services and also it is crucial to the effective achievement of patient centeredness. This finding almost consistent with the finding of studies conducted in health centers in central Ethiopia and Nigeria General Outpatient Clinics of Tertiary Hospital (16, 17, 22).

In addition, more than half $220(53 \%)$ of the respondents were given advice on how to prevent the reoccurrence of the disease. This finding is greater than the study conducted at primary health care centers in central Ethiopia, which indicate $33.3 \%$ of the respondents were given advice on how to prevent the reoccurrence of the disease (16). However, health care providers have an ethical duty to teach the patient about disease and promotion of health, as a clearly stated in the Ethiopian medical code of ethics (23). Perceived long waiting time to receive service in the hospital negatively affected patient satisfaction in this study. This finding is consistent with the studies conducted in outpatient clinics in Ethiopia (7, 17, 18, 19).

Regard to cleanliness of waiting area, $60 \%$ of the respondents was satisfied with the cleanliness of waiting area. This finding is greater than the study conducted in India Super Specialty hospital had reported that $50 \%$ of the patients were satisfied with regard to the cleanliness of the hospital (5) and also similar study was conducted in India OPD of Tertiary Care Hospital in which $55.55 \%$ of patients were satisfied with cleanliness of waiting area (24) but this finding is lower than the study conducted at selected health facilities in six regions of Ethiopia in which76.50\% (25). In the present study, nearly two third $(64.3 \%)$ of the respondents did get all prescribed drugs from the hospital pharmacy. Similar study conducted in Jimma University Specialized Hospital, Hospitals of Amhara region, Jimma hospital and Tigray Zonal hospital, indicated that $70 \%$, about $1 / 3^{\text {rd }}$ of total clients, $66.7 \%$, and $61 \%$ of the patients didn't get all or some of prescribed drugs from hospitals pharmacy respectively and lack of drugs and supplies were their major problems, which was in contrast to the findings of this study $(6,8,19,21)$. In addition, the availability of prescribed drugs from the hospital's pharmacy in this study is in line with report from a study conducted in Nepal, where it was $79 \%$ of the respondents where did gets all prescribed drugs from the hospital pharmacy (26).

Regarding to privacy during consultation, $90.7 \%$ of the respondents were satisfied with privacy during consultation. This finding is lower than the study conducted in the India Super Specialty hospital, in which $97.5 \%$ of the respondents were satisfied privacy during consultation [5], but this result is almost similar with the study conducted in Nepal OPD at Chitwan Medical College Teaching Hospital in which 91.2\% of the patients who were satisfied with the privacy during 
consultation (26).Moreover, the present study showed that the payment status as paying respondents are less satisfied as compared with non-paying (free) respondents. This may be related to their expectation of the services may rise when they incur certain costs to the services. This finding is in agreement with the studies conducted in Jimma University Specialized Hospital (6) and Eastern Ethiopia (18).

\section{Limitation of the Study}

The finding of this study might be subjected to social desirability bias because the respondents were interviewed in the hospital compound. In addition, patients may experience a relatively short-lived whereby they feel more satisfied immediately after their consultation than they do afterwards.

\section{Conclusion}

In general, the overall satisfaction of outpatient with OPD clinics health care services provided at WSUTH was low. Perceived empathy, privacy during consultation, availability of prescribed drugs, cleanliness of waiting area, cleanliness of consultation room, interviewed by their language and advice given on how to prevent the reoccurrence of disease were positively associated with patient satisfaction, but waiting time to service provider after registration process in waiting area was negatively associated. The respondent's perceptions of the providers (Physicians) empathy are of key importance in patient satisfaction. So improving provider empathy during consultation may be crucial in enhancing patient satisfaction.

\section{Recommendations}

Study Hospital management members and service providers should give attention to reduce waiting time preceding to consultation, ensure good interactions with patients, improve drugs availability and maintain privacy of patients in order to improve the level of satisfaction of patients; as well as understand the extent of the problem with cleanliness and plan to look different mechanisms to improve the cleanliness of the hospital.

Finally, it is necessary to conduct further study and periodic assessment of health services, especially from the user's satisfaction perspective.

\section{Acknowledgments}

Our special thanks and sincere appreciation go to Wolaita Sodo University and Addis Continental Institute of Public Health for sponsorship, Study participant, Data collectors, supervisors, and Wolaita Sodo University Teaching Hospital.

\section{Author's contribution}

Getu Gamo Sagaro: has conceived the study, participated in the design of the study and performed statistical analysis, and drafting the manuscript for important intellectual content.

Alemayehu Worku Yalew: has directly participated in the planning of the research, guided overall stage of the research, execution, and analysis of this study.

Mengistu Meskele Koyira: involved in design and analysis of the study, helped to draft the manuscript, drafting the article or revisiting it critically for important intellectual content:

All authors of this paper have read and approved the final version before submission.

\section{References}

[1] Donabiden A. The definition of quality and approaches to its assessment. Ann Arbor, Michigan, Health Administration Press, 1980.

[2] Bahrampour, A., and F. Zolala. Patient satisfaction and related factors in Kerman hospitals. Eastern Mediterranean Health Journal, Vol. 11, Nos 5/6, (2005) 905.

[3] Andaleeb S. Service quality perceptions and patient satisfaction: a study of hospitals in a developing country. Soc Sci Med. 2001; 52:1359-70.

[4] Ofili, A. N., and C. E. Ofovwe. Patients' assessment of efficiency of services at a teaching hospital in a developing country. Ann of Afric Med. 2005; 4(4):150-153.

[5] Jawaha, S. K. A study on outpatient satisfaction at a super specialty hospital in India. Internet Journal of Medical Update-EJOURNAL 2.2 (2007).

[6] Fekadu A., Andualem M., Yohannes H/M. Assessment of clients' satisfaction with health service deliveries at Jimma University Specialized hospital. Ethiop J Health Sci. 2011; 21(2):101-9.

[7] Asefa A, Kassa A, Dessalegn M. Patient satisfaction with outpatient health services in Hawassa University Teaching Hospital, Southern Ethiopia Journal of Public Health and Epidemiology. Vol. 6(2), pp. 101-110, February 2014.

[8] Mitike G, Mekonnen A, Osman M. Satisfaction on outpatient services in hospitals of the Amhara region. Ethiop Med J. 2002; 40:387-396.

[9] World health organization (WHO) Regional office for Africa: country cooperation strategy 2008-2011. Ethiopia: WHO 2009.

[10] World Health Organization. The World Health Report 2000. Health Systems. Improving Performance. World Health Organization Geneva Switzerland 2000.

[11] Cleary, Paul D. The increasing importance of patient surveys: Now that sound methods exist, patient surveys can facilitate improvement. BMJ: British Medical Journal 319.7212 (1999): 720.

[12] De Geyndt W: managing the quality of healthcare in developing countries, World Bank. Technical paper 1995; 258: 80 .

[13] Kane, Robert L., Matthew Maciejewski, and Michael Finch. The relationship of patient satisfaction with care and clinical outcomes. Medical care 35.7 (1997): 714-730. 
[14] McColl E, Thomas L \& Bond S (1996). A study to determine patient satisfaction with nursing care. Nursing Standard, 10(52), 34-38.

[15] Tayelgn A, Desalegn T Zegeye and Yigzaw Kebede: Mothers' satisfaction with referral hospital delivery service in Amhara Region, Ethiopia. BMC Pregnancy and Childbirth 2011, 11:78

[16] Birhanu, Zewdie, et al. Determinants of patient enablement at primary health care centers in central Ethiopia: a crosssectional study. African Journal of Primary Health Care \& Family Medicine 3.1 (2011): 8-pages.

[17] Birhanu, Zewdie, et al. Determinants of satisfaction with health care provider interactions at health centers in central Ethiopia: a cross sectional study. BMC health services research 10.1 (2010): 78 .

[18] Abdosh, Birna. The quality of hospital services in eastern Ethiopia: Patient's perspective. Ethiopian Journal of Health Development 20.3 (2006): 199.

[19] Lemessa oljira, Solomon Gebre - selassie. Satisfaction with outpatient health services at Jimma hospital, South West Ethiopia. Ethiop. J. Health Dev. 2001; 15(3); 179 - 184.

[20] Dagnew, M., and D. Zakus. Community perception on OPD performance of a teaching hospital in Gondar town. Ethiopian medical journal 35.3 (1997): 153-160.
[21] Girmay A. Assessment of clients' satisfaction with outpatient services in Tigray Zonal Hospitals. [Online]. 2006; Available from: URL:http://etd.edu.et/.

[22] Adamu, H., and M. O. Oche. Patient Satisfaction with Services at a General Outpatient Clinic of a Tertiary Hospital in Nigeria. British Journal of Medicine \& Medical Research 4.11 (2014): 2181-2202.

[23] Ethiopian Medical Association. Medical ethics for physicians practicing in Ethiopia. 2nd ed. Addis Ababa: Artistic Printing Enterprise; 2003.

[24] Patavegar BN, Shelke C, Adhav P, Kamble MS. A crosssectional study of patient's satisfaction towards services received at tertiary care hospital on OPD basis. National Journal of Community Medicine. 2012; 3(2):232-237.

[25] Bekele, Abebe, et al. Levels of outpatient satisfaction at selected health facilities in six regions of Ethiopia. Ethiopian Journal of Health Development 22.1 (2008): 42-48.

[26] Rajbanshi, L., et al. Satisfaction with health care services of outpatient department at Chitwan Medical College Teaching Hospital, Nepal. Journal of Chitwan Medical College 4.1 (2014): 11-18. 